\title{
Optical observation of the energy-momentum dispersion of spatially indirect excitons
}

\author{
A. Parlangeli, P. C. M. Christianen, and J. C. Maan \\ Research Institute for Materials, High Field Magnet Laboratory, University of Nijmegen, \\ Toernooiveld 1, 6525 ED Nijmegen, The Netherlands \\ I. V. Tokatly* \\ Lehrstuhl für Theoretische Festkörperphysik, Universität Erlangen-Nürnberg, Staudtstrasse 7/B2, 91054 Erlangen, Germany \\ C. B. Soerensen and P. E. Lindelof \\ Niels Bohr Institute, University of Copenhagen, Universitetsparken 5, DK-2100 Copenhagen, Denmark
}

(Received 5 July 2000)

\begin{abstract}
The center-of-mass $(\mathrm{CM})$ dispersion of spatially indirect excitons (IE's) in biased $\mathrm{GaAs} / \mathrm{Al}_{0.35} \mathrm{Ga}_{0.65} \mathrm{As}$ double quantum wells is determined by photoluminescence (PL) spectroscopy in an in-plane magnetic field $B$. The field rigidly shifts the IE dispersion in $k$ space by an amount proportional to both $B$ and the electron-hole separation. The PL emission arises from IE's with zero total momentum, corresponding to finite CM velocity, which allows the direct measurement of the IE CM dispersion. The observed PL energy increases quadratically in $B$, corresponding to the IE kinetic energy, whereas the PL intensity follows the thermal occupation of the optically active IE states and decreases as a Gaussian function of $B$.
\end{abstract}

The interband optical properties of semiconductors are dominated by coupled electron-hole $(e-h)$ excitationscalled excitons-that arise from the $e-h$ Coulomb interaction. ${ }^{1}$ The exciton optical spectrum consists of discrete states-similar to the Rydberg states of hydrogeneach having a dispersion that reflects the movement of the exciton as a whole. Because of the very small photon momentum $\left(\$ 10^{6} \mathrm{~m}^{-1}\right)$, optical measurements normally do not give access to excitonic states with finite center-of-mass (CM) momentum. To date, the existence of exciton dispersion has been observed only indirectly, through effects like the Fano line shape for resonant states, ${ }^{2}$ the dependence of the recombination time on the exciton mass, ${ }^{3}$ or polariton effects. ${ }^{4,5}$

In this paper, we show that the dispersion of indirect excitons (IE's) in asymmetric double quantum wells (ADQW's) can be directly measured optically by applying a magnetic field in the plane of the QW's. This unusual behavior is a consequence of the simultaneous breaking of timereversal and space-inversion symmetry in the system and can be understood by considering the usual operator of magnetic translations, which commutes with the Hamiltonian and plays the role of the $\mathrm{CM}$ momentum ${ }^{6}$

$$
\hat{\mathbf{P}}=M \hat{\mathbf{V}}-\frac{e}{c}[\mathbf{B} \times \hat{\mathbf{r}}]
$$

where $M$ is the exciton mass, $\hat{\mathbf{V}}$ is the CM velocity, and $\hat{\mathbf{r}}$ $=\hat{\mathbf{r}}_{e}-\hat{\mathbf{r}}_{h}$ is the coordinate of relative motion. The second term in Eq. (1) arises from the Lorentz force acting on the electrons and holes that constitute the exciton. A distinct consequence of Eq. (1) is that an exciton can recombine optically $(\mathbf{P} \approx 0)$ only if it has a velocity $M \mathbf{V}$ $=(e / c)[\mathbf{B} \times \mathbf{r}]$. Equation (1) is very general and applies also for bulk materials and symmetric QW's. In those systems, however, because of space-inversion symmetry, ${ }^{7}$ the expec- tation value of $\hat{\mathbf{r}}$ is zero. In contrast, for IE in an ADQW, the $e-h$ distance $d$ is almost fixed by the potential barrier; if the magnetic field lies in the plane of the QW's, the IE states shift rigidly in $k$ space in the in-plane direction perpendicular to $\mathbf{B}{ }^{8}$ The optically active states $(P \approx 0)$ correspond to IE with finite velocity, which allows the direct probe of the IE dispersion. ${ }^{9}$ Our experiment has similarities with the principle used by Hayden et al. to study the dispersion of twodimensional holes from the tunneling of emitter states in the subband minimum to states with finite $k$ in the collector. ${ }^{10}$ However, we use this principle to measure the two-particle exciton CM dispersion, which is otherwise inaccessible. This mechanism could also be used for obtaining a lowtemperature exciton gas in thermodynamic equilibrium, which may possibly lead to Bose-Einstein condensation. ${ }^{11}$ Because of the momentum shift, the in-plane magnetic field tunes the recombination lifetime of the IE's at rest to very high values, without affecting their binding energy. ${ }^{12}$

The sample studied is an ADQW made of two GaAs wells 5 and $10 \mathrm{~nm}$ wide, separated by a $5 \mathrm{~nm} \mathrm{Al}_{0.35} \mathrm{Ga}_{0.65} \mathrm{As}$ barrier. This system is optimized for the study of the IE dispersion, since the $e$ - $h$ distance $d \approx 10 \mathrm{~nm}$ is comparable to both the GaAs Bohr radius $r_{B} \sim 12 \mathrm{~nm}$ (inferring IE formation) and the magnetic length [affecting Eq. (1)] for fields of several teslas. A schematic band diagram is shown in the inset of Fig. 1(a) together with the optical transitions studied. The structure was grown in a $p-i-n$ diode and separated from the doped regions by two layers of $300 \mathrm{~nm}$ undoped $\mathrm{Al}_{0.35} \mathrm{Ga}_{0.65} \mathrm{As}$. The measurements were performed at $T$ $=4.2 \mathrm{~K}$ and in magnetic fields up to $20 \mathrm{~T}$.

In the absence of an external voltage, the optical transition with lowest energy is the direct-exciton recombination of the $10 \mathrm{~nm}$ wide well (WW). By tuning the energy levels of the two wells by the electric field $F$, the energies of the direct and the indirect transitions change, ${ }^{13,14}$ and at the field $F^{*}$ $\sim 45 \mathrm{kV} / \mathrm{cm}(\sim-2.1 \mathrm{~V}$ applied to our device), the $5 \mathrm{~nm}$ 




FIG. 1. (a) PL spectra at $B=0$ and voltages from 0 to $-3 \mathrm{~V}$. The band diagram of the sample is shown in the inset. (b) Peak positions of the direct and indirect recombination (filled and open circles, respectively). The lines are obtained from variational calculations.

narrow well $(\mathrm{NW})$ electron subband becomes the lowest in energy. At reverse biases $\lesssim-2.1 \mathrm{~V}$, therefore, the IE is the lowest-energy interband excitation. A further decrease of bias suppresses the tunneling between the wells and increases the $e-h$ distance. The photoluminescence (PL) spectra excited with a HeNe laser $\left(\sim 10^{-2} \mathrm{~W} / \mathrm{cm}^{2}\right.$, corresponding to an exciton density of $\sim 5 \times 10^{10} \mathrm{~cm}^{-2}$ ) at $B=0$ are shown in Fig. 1(a), and their peak positions are plotted in Fig. 1(b). As explained, the WW peak dominates the spectrum for $F<F^{*}$ and disappears at $F>F^{*}$, after anticrossing with the IE, which now takes all the intensity.

From the energy shift of the PL peak with bias, which is roughly proportional to the $e-h$ average distance, we estimate $d \sim 10 \mathrm{~nm}$. This value is close to the as grown distance between the centers of the QW's and consistent with all the measurements. We determined the IE binding energy, using variational calculations [solid curve in Fig. 1(b)] that take into account excitonic effects ${ }^{15}$ and found a binding energy of $9 \mathrm{meV}$ for the direct exciton and $3.5 \mathrm{meV}$ for the indirect one, in fair agreement with our data of the Stark shift [Fig. 1(b)]. When the bias is increased, the total IE PL intensity decreases because of the suppression of the $e-h$ overlap, which eventually leads to such a long optical recombination time $^{12}$ that nonradiative recombination channels take over.

The IE spectra measured in a magnetic field are shown in Fig. 2(a) for a bias of $-4 \mathrm{~V}(70 \mathrm{kV} / \mathrm{cm})$. The IE PL is quenched by fields of $\sim 5 \mathrm{~T}$ and decays as a Gaussian function of the magnetic field, as shown in Fig. 3(a) (circles). In addition, we show in Fig. 2(b) the IE shift, which is quadratic in field and amounts to $0.021 \mathrm{meV} / \mathrm{T}^{2} \times B^{2}$. We measured the effect of $e-h$ separation on the PL intensity for different voltages, and the results in Fig. 3(a) show that at higher bias (i.e., larger $d$ ) the PL is suppressed by smaller magnetic fields. The diamagnetic shift of the WW exciton was found to be only of $\sim 0.005 \mathrm{meV} / \mathrm{T}^{2} \times \mathrm{B}^{2}$. We stress that these data are qualitatively different from those measured

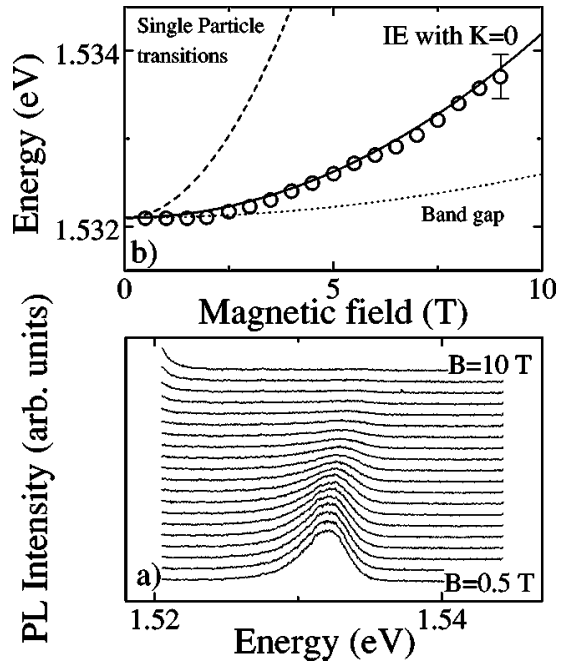

FIG. 2. (a) IE PL spectra at $-4 \mathrm{~V}(70 \mathrm{kV} / \mathrm{cm})$ for magnetic fields ranging from 0.5 to $10 \mathrm{~T}$, with $0.5 \mathrm{~T}$ step. The peak is suppressed by fields $B \sim 5 \mathrm{~T}$ (Fig. 3). (b) Measured quadratic shift of the indirect exciton (symbols). The error bar is shown on one data point. The measured value of $0.021 \mathrm{meV} / \mathrm{T}^{2}$ agrees with the model proposed in the text (line). The energy of the fundamental gap and of the single particle recombination are plotted for comparison with dotted and dashed lines, respectively.

with a magnetic field perpendicular to the QW and, as we will show below, they directly give information on the IE CM motion.

The basic features of our experiment can be understood from Eq. (1). In the system, $d$ is primarily fixed by the confining potential and only slightly depends on the magnetic field and the CM momentum. The expectation value of Eq. (1) gives thus an explicit expression of the $\mathrm{CM}$ velocity $\mathbf{V}$ in terms of CM momentum $\mathbf{P}$, and we can obtain the IE dispersion law $\varepsilon_{i d}(\mathbf{P})$ by direct integration


FIG. 3. (a) Luminescence intensity of the indirect excitons (IE's) at different biases as a function of magnetic field. The data are normalized to the intensity at $B=0.5 \mathrm{~T}$. (b) Boltzmann factor of the optically active IE. The voltages $-2.5,-3$, and $-4 \mathrm{~V}$, correspond to $d=7.7,9.1$, and $9.7 \mathrm{~nm}$, respectively (see text). The function at $-5 \mathrm{~V}(9.9 \mathrm{~nm})$ is indistinguishable from the one at $-4 \mathrm{~V}$. 


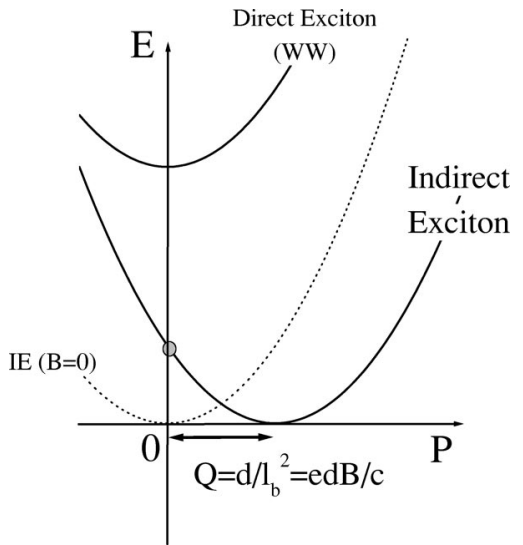

FIG. 4. Effect of the in-plane magnetic field on the exciton dispersion, which is characterized by a mass $M=m_{e}+m_{h}$ (see text). The direct exciton (wide well) remains unaffected, whereas the indirect exciton dispersion shifts in $k$ space by an amount $Q$ $=e d B / c=d / l_{b}^{2}$. The optically active IE's $(P \approx 0)$ are indicated with a circle.

$$
\varepsilon_{i d}(\mathbf{P})=E_{i d}+\frac{1}{2 M}\left(\mathbf{P}+\frac{e}{c}[\mathbf{B} \times \mathbf{d}]\right)^{2},
$$

where $E_{i d}$ is the energy of IE at rest, and $M$ is the total mass of the exciton, given by the sum of the electron and hole effective masses. As shown in Fig. 4, the effect of the magnetic field is to rigidly shift the IE dispersion in $k$ space by $Q=e d B / c=d / l_{B}^{2}$, where $l_{B}$ is the magnetic length. The optically active IE's $(P \approx 0)$ correspond to a nonzero $C M$ velocity $V=e d B / M c$ in the direction perpendicular to both the magnetic field and the growth axis. Therefore, the PL measurements allow the direct study of the IE dispersion relation $\varepsilon_{i d}(Q)=Q^{2} / 2 M$. The IE shift, i.e., the shift of the PL energy, is simply equal to the kinetic energy $\varepsilon_{i d}$ $=e^{2} d^{2} B^{2} / 2 M c^{2} \equiv a B^{2}$, and the IE thermal occupation is proportional to the Boltzmann factor $e^{-a B^{2} / K_{B} T}\left(K_{B}\right.$ is the Boltzmann constant), which is a Gaussian function of $B$.

This simple picture quantitatively describes our data of the quadratic shift of the PL energy and the Gaussian decay of the PL intensity with field, which are both described by the single parameter $a$. In Fig. 2(b), we compare the experimental parabolic shift at $-4 \mathrm{~V}$ with the calculated kinetic energy of the IE with $P=0$. Using $d=10 \mathrm{~nm}$ and $M=m_{e}$ $+m_{h}\left(m_{e}=0.0665 m_{0}\right.$ and $m_{h}=0.35 m_{0} . m_{0}$ is the freeelectron mass), we found $a=0.021 \mathrm{meV} / \mathrm{T}^{2}$ [solid curve in Fig. 2(b)], in excellent agreement with the experiment. Furthermore, the PL intensity follows the thermal occupation of the $P=0$ states and indeed decays as a Gaussian characterized by a value of $a$ that equals the one obtained from the PL shift. We stress that two independent determinations of the same parameter $a$ yield precisely the same value, which strongly supports our interpretation.

IE recombination is the only mechanism that can explain the observed field dependence and all other conceivable processes can be excluded. To illustrate this point, we show in Fig. 2(b) the calculated diamagnetic shifts of the optically allowed $(\Delta k=0)$ transition of free $e-h$ pairs (dashed lines) and the $\Delta k \neq 0$ optically forbidden band-edge transition (dotted line). The shift of the $e-h$ recombination is an order of magnitude larger, as observed by Whittaker et al., ${ }^{16}$ whereas the shift of the band-edge, which is comparable to the measured shift of the WW direct exciton (not shown), is four times too small. Strictly speaking, the small shift of the band edge $^{17}$ should be included in the observed shift, but this would lead to a correction smalller than the experimental accuracy [Fig. 2(b)].

Further experimental proof comes from the measurements of the bias dependence shown in Fig. 3(a). Upon changing bias from resonance to the IE regime, the electron wave function changes from being delocalized in the two wells to being well confined in the NW. Since the holes remain always well confined in the WW, the electron localization strongly affects the $e-h$ distance, which changes from $\sim 5$ $\mathrm{nm}$ at anticrossing to $\sim 10 \mathrm{~nm}$ at high reverse bias $(\lessgtr-4 \mathrm{~V})$. The change of $d$ affects the shift in momentum described by Eqs. (1) and (2), which is in turn observed in the PL intensity [Fig. 3(a)].

To model this behavior schematically, we consider an idealized two-level system, in which purely direct $(d=0)$ and purely indirect $\left(d=d_{0}=10 \mathrm{~nm}\right)$ excitons are coupled by tunneling, which mixes these two states and allows solutions with intermediate $d$. Only the electrons participate in the tunneling process, and we thus estimate $d$ from the probability of the purely indirect state, i.e., from the probability $u^{2}$ to find an electron in the NW:

$$
d=d_{0} u^{2}=\frac{d_{0}}{2}\left(1+\frac{\sqrt{\Delta E^{2}-4 t^{2}}}{\Delta E}\right),
$$

where $t$ is the tunneling matrix element (independent from bias and magnetic field), and $\Delta E$ is the energy difference between the eigenvalues of the two-level Hamiltonian.

By using $t \approx 2.5 \mathrm{meV}$ (from the splitting between the direct and indirect excitons at the anticrossing) and $\Delta E$ as the difference between the measured IE energy and the calculated one for the direct excitons [Fig. 1(b)], we obtained $d$ as a function of bias. We found $d=7.7,9.1,9.7$, and $9.9 \mathrm{~nm}$ for $-2.5,-3,-4$, and $-5 \mathrm{~V}$, respectively. From these values, we obtained the coefficient $a(d)=e^{2} d^{2} / 2 M c^{2}$, which enters in the Boltzmann population $\left(e^{-a B^{2} / K_{B} T}\right)$ of the $P=0$ IE. When $d$ increases with bias, the number of thermally excited excitons thus decreases more rapidly with magnetic field. The calculated magnetic-field dependence of the Boltzmann population of the $P=0 \mathrm{IE}$ is plotted in Fig. 3(b) for the considered biases (the curve at $-5 \mathrm{~V}$, indistinguishable from the one at $-4 \mathrm{~V}$, is omitted). The agreement with the experiment [Fig. 3(a)] is remarkably good, and confirms the validity of our model.

We stress that our results cannot be explained by free $e-h$ recombination, since this would not depend on $d / l_{B}^{2}$ as for the IE. Instead, the free-carrier recombination would be more strongly suppressed at lower reverse bias-when the electrons are less localized in the NW-than at higher reverse bias, which is opposite to the experimental trend [Fig. 3(a)].

In conclusion, we have shown that the PL of IE in a biased ADQW in the presence of in-plane magnetic fields can be used to determine the IE CM dispersion [Eq. (2)]. This unique possibility arises from the shift of the IE disper- 
sion in $k$ space by an amount $Q=e d B / c$, which makes it possible to observe the PL of states that are otherwise optically forbidden. We can describe quantitatively the magnetic-field dependence of the IE PL intensity and peak position, as well as the behavior as a function of bias, i.e., $e-h$ spatial separation. We believe that our experiments are the first direct demonstration of the fundamental relation (1), which arises from the simultaneous breaking of spaceinversion and time-reversal symmetry in the system.
This work is part of a research program of the Stichting voor Fundamenteel Onderzoek der Materie (FOM) financially supported by NWO (The Netherlands), and it has been supported also by the European Commission under Contract No. ERBFMGECT950079. The samples were grown at the III-V NANOLAB of the MIC and NBI (Denmark). The work of I.T. was supported by the Alexander von Humboldt Foundation. We are grateful to A. B. Dzyubenko and A. Fasolino for useful discussions.
*On leave from Moscow Institute of Electronic Engineering, Zelenograd, 103498, Russia.

${ }^{1}$ F. Bassani and G. Pastori Parravicini, Electronic States and Optical Transitions in Solids (Pergamon Press, Oxford, 1975).

${ }^{2}$ D. Y. Oberli et al., Phys. Rev. B 49, 5757 (1994).

${ }^{3}$ J. Feldmann et al., Phys. Rev. Lett. 59, 2337 (1987).

${ }^{4}$ R. Houdré et al., Phys. Rev. Lett. 73, 2043 (1994).

${ }^{5}$ A. Tredicucci et al., Phys. Rev. Lett. 75, 3906 (1995).

${ }^{6}$ L. P. Gor'kov and I. E. Dzyaloshinskii, Zh. Éksp. Teor. Fiz. 53, 717 (1967) [Sov. Phys. JETP 26, 449 (1968)]; J. E. Avron, I. W. Herbst, and B. Simon, Ann. Phys. 114, 431 (1978).

${ }^{7}$ Strictly speaking, we refer to the symmetry of the excitonic envelope function in the ground state.

${ }^{8}$ A. A. Gorbatsevich and I. V. Tokatly, Semicond. Sci. Technol. 13, 288 (1998).

${ }^{9}$ This technique can also be potentially used to probe the exciton roton minimum predicted at small $e-h$ distances by Yu. E. Lozovik and A. M. Ruvinsky, Phys. Lett. A 227, 271 (1997).

${ }^{10}$ R. K. Hayden et al., Phys. 46, 15586 (1992).

${ }^{11}$ L. V. Butov and A. I. Filin, Phys. Rev. B 58, 1980 (1998), and references therein.

${ }^{12}$ S. Charbonneau et al., Phys. Rev. B 38, 6287 (1988).

${ }^{13}$ Y. J. Chen et al., Phys. Rev. B 36,4562 (1991).

${ }^{14}$ C. H. Perry et al., J. Luminescence 48-49, 725 (1991).

${ }^{15}$ A. M. Fox et al., Phys. Rev. B 44, 6231 (1991).

${ }^{16}$ D. M. Whittaker, T. A. Fisher, P. E. Simmonds, M. S. Skolnick, and R. S. Smith, Phys. Rev. Lett. 67, 887 (1991).

${ }^{17}$ The observed diamagnetic shift of the WW exciton $(0.005$ $\mathrm{meV} / \mathrm{T}^{2}$-see text) can be taken as a reference. The IE diamagnetic shift would be slightly smaller, since the electron states in the IE regime are localized in the NW. 